\title{
Analyses of associations with asthma in four asthma population samples from Canada and Australia
}

\author{
Denise Daley $\cdot$ Mathieu Lemire $\cdot$ Loubna Akhabir $\cdot$ Moira Chan-Yeung · Jian Qing He \\ Treena McDonald · Andrew Sandford · Dorota Stefanowicz • Ben Tripp · David Zamar • \\ Yohan Bosse · Vincent Ferretti - Alexandre Montpetit · Marie-Catherine Tessier · Allan Becker • \\ Anita L. Kozyrskyj · John Beilby · Pamela A. McCaskie · Bill Musk · Nicole Warrington • \\ Alan James · Catherine Laprise - Lyle J. Palmer · Peter D. Paré · Thomas J. Hudson
}

Received: 30 November 2008/ Accepted: 13 February 2009/Published online: 27 February 2009

(C) Springer-Verlag 2009

\begin{abstract}
Asthma, atopy, and related phenotypes are heterogeneous complex traits, with both genetic and environmental risk factors. Extensive research has been conducted and over hundred genes have been associated with asthma and atopy phenotypes, but many of these findings have failed to replicate in subsequent studies. To separate true associations from false positives, candidate
\end{abstract}

D. Daley and M. Lemire contributed equally to this manuscript.

Electronic supplementary material The online version of this article (doi:10.1007/s00439-009-0643-8) contains supplementary material, which is available to authorized users.

D. Daley · L. Akhabir · J. Q. He · T. McDonald .

A. Sandford · D. Stefanowicz · B. Tripp .

D. Zamar · P. D. Paré (ه)

James Hogg iCAPTURE Center,

University of British Columbia (UBC),

1081 Burrard Street, Vancouver, BC V6Z 1Y6, Canada

e-mail: ppare@mrl.ubc.ca

M. Lemire · V. Ferretti - A. Montpetit - M.-C. Tessier •

T. J. Hudson

McGill University and Génome Québec Innovation Centre,

Montreal, QC, Canada

M. Lemire · V. Ferretti · T. J. Hudson

Ontario Institute for Cancer Research,

Toronto, ON, Canada

\section{Chan-Yeung}

Occupational and Environmental Lung Disease Unit,

University of British Columbia,

Vancouver, BC, Canada

\section{Y. Bosse}

Centre de Recherche, Hôpital Laval, Institut Universitaire de Cardiologie et de Pneumologie de l'Université Laval,

Quebec, Canada genes need to be examined in large well-characterized samples, using standardized designs (genotyping, phenotyping and analysis). In an attempt to replicate previous associations we amalgamated the power and resources of four studies and genotyped 5,565 individuals to conduct a genetic association study of 93 previously associated candidate genes for asthma and related phenotypes using the same set of 861 single-nucleotide polymorphisms (SNPs), a common genotyping platform, and relatively harmonized phenotypes. We tested for association between SNPs and the dichotomous outcomes of asthma, atopy, atopic asthma, and airway hyperresponsiveness using a general allelic

Y. Bosse

Laval University Hospital Research Center (CRCHUL), Quebec, Canada

A. Becker - A. L. Kozyrskyj

Department of Pediatrics and Child Health,

Faculty of Medicine, University of Manitoba, Winnipeg,

Manitoba, Canada

A. L. Kozyrskyj

Faculty of Pharmacy, Manitoba Center for Health Policy,

Winnipeg, Manitoba, Canada

A. L. Kozyrskyj

Department of Community Health Sciences,

Manitoba Center for Health Policy, Winnipeg,

Manitoba, Canada

J. Beilby · B. Musk · A. James

Sir Charles Gairdner Hospital, Perth, WA, Australia

P. A. McCaskie - N. Warrington - L. J. Palmer

Laboratory for Genetic Epidemiology, Western Australian

Institute for Medical Research, UWA Centre for Medical

Research and School of Population Health,

University of Western Australia,

Perth, Australia 
likelihood ratio test. No SNP in any gene reached significance levels that survived correction for all tested SNPs, phenotypes, and genes. Even after relaxing the usual stringent multiple testing corrections by performing a genebased analysis (one gene at a time as if no other genes were typed) and by stratifying SNPs based on their prior evidence of association, no genes gave strong evidence of replication. There was weak evidence to implicate the following: IL13, IFNGR2, EDN1, and VDR in asthma; IL18, TBXA2R, IFNGR2, and VDR in atopy; TLR9, $T B X A 2 R, V D R, N O D 2$, and STAT6 in airway hyperresponsiveness; TLR10, IFNGR2, STAT6, VDR, and NPSR1 in atopic asthma. Additionally we found an excess of SNPs with small effect sizes $(\mathrm{OR}<1.4)$. The low rate of replication may be due to small effect size, differences in phenotypic definition, differential environmental effects, and/or genetic heterogeneity. To aid in future replication studies of asthma genes a comprehensive database was compiled and is available to the scientific community at http://genapha.icapture.ubc.ca/.

\section{Introduction}

Studies of twins, families, and birth cohorts have all shown that genetics plays an important role in the development of asthma, but it has been difficult to clearly identify the exact genetic variants involved (Ober and Hoffjan 2006). There is no shortage of positive findings and candidate genes, but replication has proved difficult, and even the most replicated associations have one or more negative reports (Ober and Hoffjan 2006). There is a clear need for well designed and executed replication studies.

The aim of this study was to identify by rigorous replication the most likely candidate genes for asthma and related phenotypes, based on a common-disease, commonvariant hypothesis. According to this hypothesis, genetic variants that impart risk for common diseases will have common alleles that are present in all populations and be associated with a relatively modest effect size (Blakey et al. 2005; Chanock et al. 2007). Large sample sizes consisting of thousands of subjects are needed to clearly define the contribution of these variants to asthma and allergic phenotypes (Blakey et al. 2005; Chanock et al. 2007; Maier et al. 2006).

C. Laprise

Département des sciences fondamentales, Université du Québec à Chicoutimi, Saguenay, Quebec, Canada

C. Laprise

Université de Montréal Community Genomic Medicine Center, Chicoutimi Hospital, Saguenay, Quebec, Canada
The present study design involved testing genetic variants in 93 genes, previously reported (in one or more studies) to be associated with asthma and/or related traits (Supplementary Table S1), in four panels that include three family based studies and a population-based, case-control sample (Table 1). These combined resources have sufficient power $(\geq 50 \%)$ to detect a spectrum of alleles with heterozygote relative risk (RR) $>1.5$ (Fig. 1).

Four discrete phenotypes (asthma, atopy, atopic asthma, and airway hyperresponsiveness (AHR)) were selected a priori and tested for association using a general allelic likelihood ratio test. We have used physician diagnosis (asthma), skin prick tests (atopy), and results of methacholine challenge (AHR) to define both affected and unaffected individuals (see "Methods"). We harmonized (asthma, atopic asthma, and AHR) and standardized (atopy) phenotype definitions between panels.

We selected 154 SNPs with prior evidence for association (positive and negative) with one or more asthmarelated phenotypes and we supplemented these with 26 coding non-synonymous SNPs in previously associated genes. Selected SNPs had a minor allele frequency $(\mathrm{MAF}) \geq 0.05$ in at least one HapMap population. We complemented the study with 719 tagSNPs, which were within gene-intervals that include $10 \mathrm{~kb}$ sequences, both upstream and downstream, as determined by using NCBI Build \#34 of the human genome reference sequence (see "Methods").

Despite the relatively large sample size few (13\%) of the genes showed any evidence for association with one or more phenotype. This study which represents the most comprehensive attempt to date at replication of genetic associations in asthma/allergy was conducted at the dawn of the era of genome wide association studies which are sure to identify additional candidates and illustrates the need to carefully re-evaluate positive associations in large and heterogeneous populations.

\section{Methods}

All DNA samples were collected with informed consent, in compliance with each recruiting center's Research Ethics Boards.

Study samples (panels)

The individual panels include (1) the Canadian Asthma Primary Prevention Study (CAPPS) cohort (Becker et al. 1999, 2004; Chan-Yeung et al. 1999, 2000, 2005; He et al. 2003; Hegele et al. 2001; Kaan et al. 2000), which is comprised of 549 children from 545 families at high risk for developing asthma and their parents and is predominantly 
Table 1 Panel sizes by study, phenotype and ethnic background

\begin{tabular}{|c|c|c|c|c|c|c|c|c|c|}
\hline \multirow[b]{2}{*}{ Sample } & \multicolumn{4}{|c|}{ Family based trios } & \multicolumn{2}{|c|}{ Case-control } & \multicolumn{2}{|c|}{ Combined } & \\
\hline & CAPPS & SAGE & SLSJ & Total & \multicolumn{2}{|c|}{ Busselton } & \multirow{2}{*}{\multicolumn{2}{|c|}{ 1,574 families/trios }} & \\
\hline Families & 545 & 723 & 306 & 1,574 & \multicolumn{2}{|c|}{ 1,549 individuals } & & & \\
\hline Genotyped & 1,316 & 1,466 & 1,234 & 4,016 & \multicolumn{2}{|c|}{ 1,549 individuals } & \multicolumn{2}{|c|}{5,565 individuals } & \\
\hline Phenotype & $\begin{array}{l}\text { Complete } \\
\text { trios }\end{array}$ & $\begin{array}{l}\text { Complete } \\
\text { trios }\end{array}$ & $\begin{array}{l}\text { Complete } \\
\text { trios }\end{array}$ & & $\begin{array}{l}\text { ed } \\
\text { e trios }\end{array}$ & Cases & Controls & Total & $\begin{array}{l}\text { Total case } \\
\text { control and } \\
\text { complete trios }\end{array}$ \\
\hline \multicolumn{10}{|c|}{ Caucasian samples } \\
\hline Asthma & 51 & 109 & 379 & 53 & & 644 & 751 & 1,395 & 1,934 \\
\hline Atopy & 105 & 145 & 362 & 61 & & 620 & $575^{\mathrm{a}}$ & 1,195 & 1,807 \\
\hline AHR & 142 & 96 & 278 & 51 & & 213 & $814^{\mathrm{b}}$ & 1,027 & 1,543 \\
\hline Atopic asthma & 37 & 71 & 305 & 41 & & 382 & $400^{\mathrm{c}}$ & 782 & 1,195 \\
\hline \multicolumn{10}{|c|}{ Non Caucasian samples ${ }^{\mathrm{d}}$} \\
\hline Asthma & 3 & 28 & $\mathrm{n} / \mathrm{a}$ & 31 & & $\mathrm{n} / \mathrm{a}$ & $\mathrm{n} / \mathrm{a}$ & $\mathrm{n} / \mathrm{a}$ & 31 \\
\hline Atopy & 18 & 44 & $\mathrm{n} / \mathrm{a}$ & 62 & & $\mathrm{n} / \mathrm{a}$ & $\mathrm{n} / \mathrm{a}$ & $\mathrm{n} / \mathrm{a}$ & 62 \\
\hline AHR & 14 & 22 & $\mathrm{n} / \mathrm{a}$ & 36 & & $\mathrm{n} / \mathrm{a}$ & $\mathrm{n} / \mathrm{a}$ & $\mathrm{n} / \mathrm{a}$ & 36 \\
\hline Atopic asthma & 3 & 20 & $\mathrm{n} / \mathrm{a}$ & 23 & & $\mathrm{n} / \mathrm{a}$ & $\mathrm{n} / \mathrm{a}$ & $\mathrm{n} / \mathrm{a}$ & 23 \\
\hline \multicolumn{10}{|c|}{ Combined analysis (complete trios and case-control samples from four studies) ${ }^{\mathrm{e}}$} \\
\hline Asthma & 57 & 139 & 379 & 57 & & 644 & 751 & 1,395 & 1,970 \\
\hline Atopy & 135 & 190 & 362 & 68 & & 620 & 575 & 1,195 & 1,882 \\
\hline AHR & 170 & 120 & 278 & 56 & & 213 & 814 & 1,027 & 1,595 \\
\hline Atopic asthma & 43 & 92 & 305 & 44 & & 382 & 400 & 782 & 1,222 \\
\hline
\end{tabular}

${ }^{a}$ Controls may include asthma cases

b Controls may include asthma cases

${ }^{c}$ Controls must not have asthma or atopy

${ }^{\text {d }}$ Sample is Asian (China, Korea, Japan) and Canadian First Nation descent

e Combined analysis also contains trio's with mixed and unknown ethnicity

Fig. 1 Power of the combined samples, on the $y$-axes, to detect an association with asthma status at $P<0.00001$, for a susceptibility allele found in frequency q in the population, having a genetic effect represented by the relative risk on the $x$-axis. Each curve is associated with a different risk allele frequency q, as indicated on the graphs. In the left panel, the minor allele of a SNP is the risk allele; in the right panel, the major allele of a SNP is the risk allele. Each point on the power curves is estimated from 1,000 simulated replicates, assuming a disease prevalence of $7.5 \%$, an additive model for the risk and homogeneity across samples

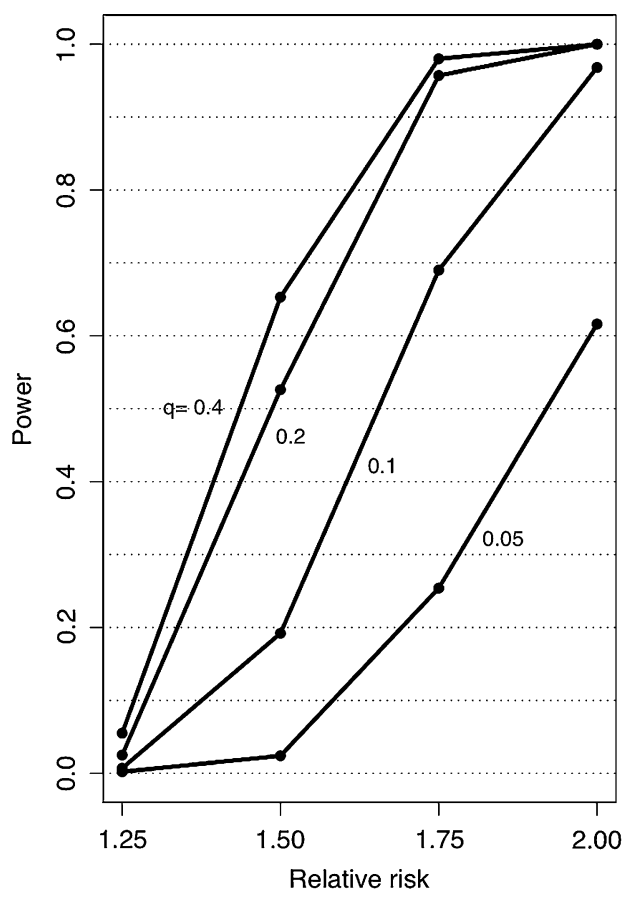


Caucasian (79.2\%); (2) the Study of Asthma Genes and Environment (SAGE) cohort (Kozyrskyj et al. 2008; Mai et al. 2007) composed of 723 children and their parents from Manitoba, Canada, who are primarily Caucasian (74.2\%); (3) the Saguenay-Lac-Saint-Jean and Quebec City Familial Asthma Collection (SLSJ) (Begin et al. 2007; Laitinen et al. 2004; Laprise et al. 2004; Poon et al. 2004, 2005; Raby et al. 2002; Tremblay et al. 2006) consisting of a French-Canadian founder population panel of 306 multigenerational families with at least one asthmatic proband; and (4) The Busselton Health Study (Busselton) (James et al. 2005a, b), a population-based, nested, case-control panel of 1,549 individuals of European Caucasian descent from Australia. Of note is that at the time of phenotyping, subjects in the CAPPS and SAGE panels were between the ages of 6 and 8 (birth cohorts), whereas subjects in the SLSJ and Busselton panels were primarily teenagers or adults. Detailed information regarding each study panel is provided in the online supplement.

\section{Phenotype definitions}

Four dichotomous phenotypes were defined a priori for the analysis:

(1) asthma defined as doctor-diagnosed asthma in CAPPS, SAGE, and SLSJ, and as a positive response to the question "has a doctor ever told you that you had asthma, or bronchial asthma?" at either survey (1981 and 1994) in the Busselton panel. Detailed information regarding the asthma phenotype is contained in the online supplement. (Supplementary Table S2)

(2) atopy defined as any skin-prick test wheal at least $3 \mathrm{~mm}$ greater than the negative control for any allergen tested (Supplementary Table S2 for specific allergens);

(3) atopic asthma defined as individuals diagnosed with both asthma and atopy; and

(4) airway hyperresponsiveness as assessed by methacholine-challenge testing in all four samples. A positive response was defined as a $20 \%$ decrease in $\mathrm{FEV}_{1}$. For the SLSJ sample, AHR was defined as $\mathrm{PC}_{20}<8 \mathrm{mg} / \mathrm{ml}$; for Busselton, $\mathrm{PD}_{20} \leq 3.9 \mu \mathrm{mol}$. Because AHR is more prevalent in children (Godfrey 2000; Godfrey et al. 1999), for the childhood samples (CAPPS \& SAGE), we used $\mathrm{PC}_{20}<3.2 \mathrm{mg} / \mathrm{ml}$, because it yields the greatest sum of sensitivity and specificity in children (Godfrey 2000).

Gene and SNP selection

For this study, we selected genes that were reported to be associated with asthma or related phenotypes in at least one prior study. We selected 154 SNPs that had been reported in these studies, and supplemented these with 26 coding non-synonymous SNPs in these candidates reported in dbSNPs (build 34) that had frequency data and a MAF $>0.05$ in any population, and 719 tagging SNPs that were selected from HapMap (2005), or SeattleSNPs (see web resources in Appendix), or Innate Immunity (see web resources in Appendix) of the National Heart Lung and Blood Institute's Programs for Genomic Applications by using linkage disequilibrium data derived from the HapMap CEU dataset.

\section{Genotyping and data cleaning}

We genotyped samples with the Illumina Bead Array System in accordance with the manufacturer's protocol (Shen et al. 2005) and according to Lincoln et al. (2005). Fourteen SNPs were genotyped using TaqMan assays (Applied Biosystems) (see Supplementary Methods). For 669 samples from SAGE and CAPPS, we used DNA templates generated using a Whole Genome Amplification method (WGA), using the RepliG Midi kit (Qiagen Cat\# 150045) and the protocol described by (Qiagen, Hilden, Germany). We retained markers for analysis if they had a minimum call rate of $90 \%$, a maximum of four Mendelian errors, a maximum of one reproducibility error, and showed consistency with Hardy-Weinberg equilibrium at the level $P>0.001$. In total, we genotyped $898 \mathrm{SNPs}$, of which 861 passed all quality control checks and entered the analysis.

\section{Statistical analyses}

We tested for association between SNPs and the dichotomous outcomes of (asthma, atopy, atopic asthma, and airway hyperresponsiveness) using a general allelic likelihood ratio test $\chi^{2}$ (one degree of freedom) as implemented in UNPHASED (Dudbridge 2003, 2008) (see online supplement for details). Our strategy for correction for multiple testing was influenced by the study design. If we were to apply a Bonferroni correction, there would be no SNP that would survive this stringent level of correction. However, it is recognized that a Bonferroni correction does not take into account the correlation between tagSNPs, and would result in a significant overcorrection and subsequent loss of power (Nyholt 2004). This is a large scale replication study, with one of the aims being to rank order the significance of genes to the etiology of asthma. Some genes needed more SNPs to be adequately covered than others. Applying a global multiple correction factor, in this study to each individual SNP result would have an undesirable effect: densely typed genes would show greater trends of association merely because they use a greater proportion of the total 
SNP resources. Rather than correcting for all genes and SNPs tested we employed a gene-based approach by applying a correction only with respect to the number of SNPs in that gene and its neighborhood and the number of phenotypes tested (Nyholt 2004). For each gene investigated, an effective number of independent SNPs was calculated by using the definition of $\mathrm{Li}$ and $\mathrm{Ji}$ (2005), as implemented in SNPSpD (Nyholt 2004). By using a similar procedure, the Matrix Spectral Decomposition (matSpD) approach, we estimated the effective number of independent phenotypes.

We recognize some readers may not be satisfied that this computationally fast approach adequately approximates the permutation distribution. Therefore, when a SNP was deemed significant using this fast approach, the corrected significance levels (cP) were corroborated using the computationally intensive permutation procedure implemented in UNPHASED (Permutation cP), using 50,000 random permutations for each genes.

This correction strategy was deemed to be appropriate for tagSNPs, which were selected for indirect tests of association, the hypothesis being that the marker SNP is only in LD with an unknown disease variant. The prior probability for a SNP to be associated with an asthma related phenotype differs between a tagSNP and a SNP previously reported to be associated with our phenotypes of interest. Thus, there is a natural stratification of the prior probability for association in our dataset. We choose to account for this difference in priors, using a simple stratified approach. We grouped our SNPs into two strata's, one with SNPs identified in the literature with at least one prior positive association with an asthma related phenotype $(N=104)$, and the second strata containing the remaining SNPs. For SNPs in the former strata, we conducted a direct test of association, whereby we corrected the nominal $P$ value only for the number of phenotypes evaluated in the study.

We present the nominal $P$ value, the corrected (cP), and the permutation $P$ value where appropriate (Tables 2 and 3). We also supply the published references for SNPs previously reported in the literature, allowing the reader to fully evaluate the evidence for statistical significance and association. Additionally, for the 104 SNPs that were not subjected to a full correction we provide both the $P$ values for both the modified and standard correction strategy. This allows the reader to select the level of significance they feel is appropriate in light of the prior evidence for association.

Website and online database

(http://genapha.icapture.ubc.ca/)

To support further identification and research into the most robust candidate genes for asthma and related phenotypes, we have compiled a comprehensive database that is being made available to the scientific community on our website (http://genapha.icapture.ubc.ca/). This database contains information on all genes and SNPs studied in the four panels and includes detailed information on allele frequencies (with comparision to HapMap frequencies), association results, corrected and uncorrected $P$ values, and linkage disequilibrium (LD) plots for each gene by sample. Search tools have been created and incorporated into the database to allow users to search and identify information of interest to them. Users can search by gene or by SNP. For each SNP there is a comprehensive summary page that provides the following information; SNP function (intron, exon, non-synonymous and synonymous coding), detailed association results for all panels and phenotypes for that SNP, links to dbSNPS, OMIM, NCBI, The Genetic Association Database, Pharm GKB, UCSC Genome Browser, Seattle SNPs, Innate Immunity and KEGG databases. A pathway search interfaces our results with the KEGG database, users can search by gene, SNP, or genetic pathway. For each search a list of all genetic pathways identified in the KEGG database is displayed. Once a pathway has been selected a pathway graphic is displayed and the genes which have been genotyped in our datasets are highlighted and appear in a box on the left hand side of the screen. Users can then view association results for genes in specific genetic pathways of interest. Exhaustive literature searches have been conducted to identify previous genetic associations with allergic disease phenotypes. If a SNP has been previously reported in the literature this is identified on the SNP summary page (* Previous Associations) and clicking on the link provides users with a reference list. A full tutorial (http://genapha.icapture.ubc. ca/research-tutorial.do) is provided to facilitate the access and use of this unique and valuable resource by the asthma and allergy community.

\section{Results}

Genotyping and data cleaning

After applying standard quality filters to the set of 898 SNPs (see "Methods"), we retained 861 SNPs for analysis. From a set of 372 replicate samples, we observed only 20 genotype differences, resulting in a discrepancy rate of $0.005 \%$. In the family based samples, relationships between samples were confirmed by comparing pairwise average number of alleles identical by state to what is expected. A total of 24 families (13 CAPPS, 8 SAGE, and 3 SLSJ) were excluded due to either mispaternity or unresolved DNA switches. At most one Mendelian inconsistency was observed for $99.1 \%$ of the SNPs in the 


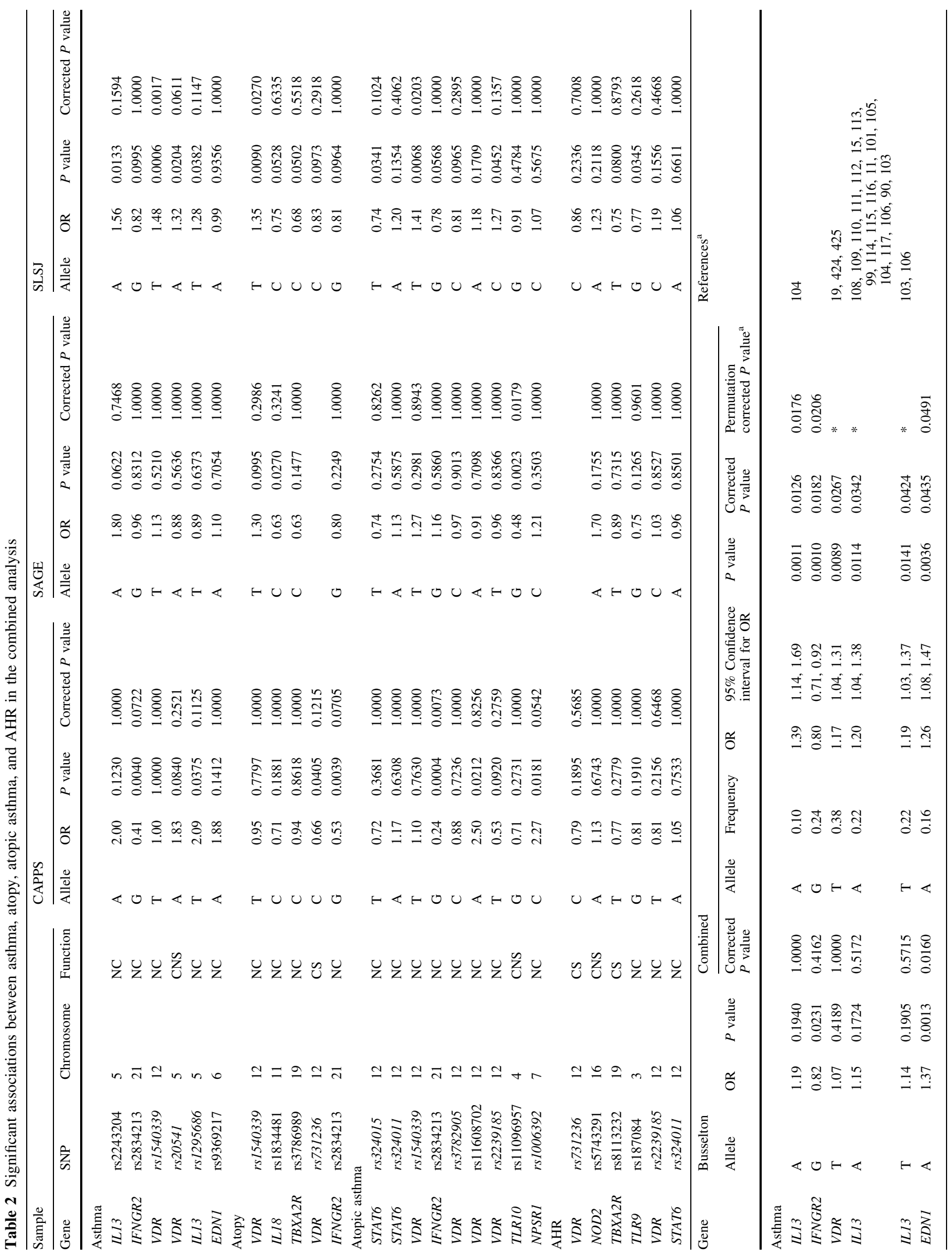




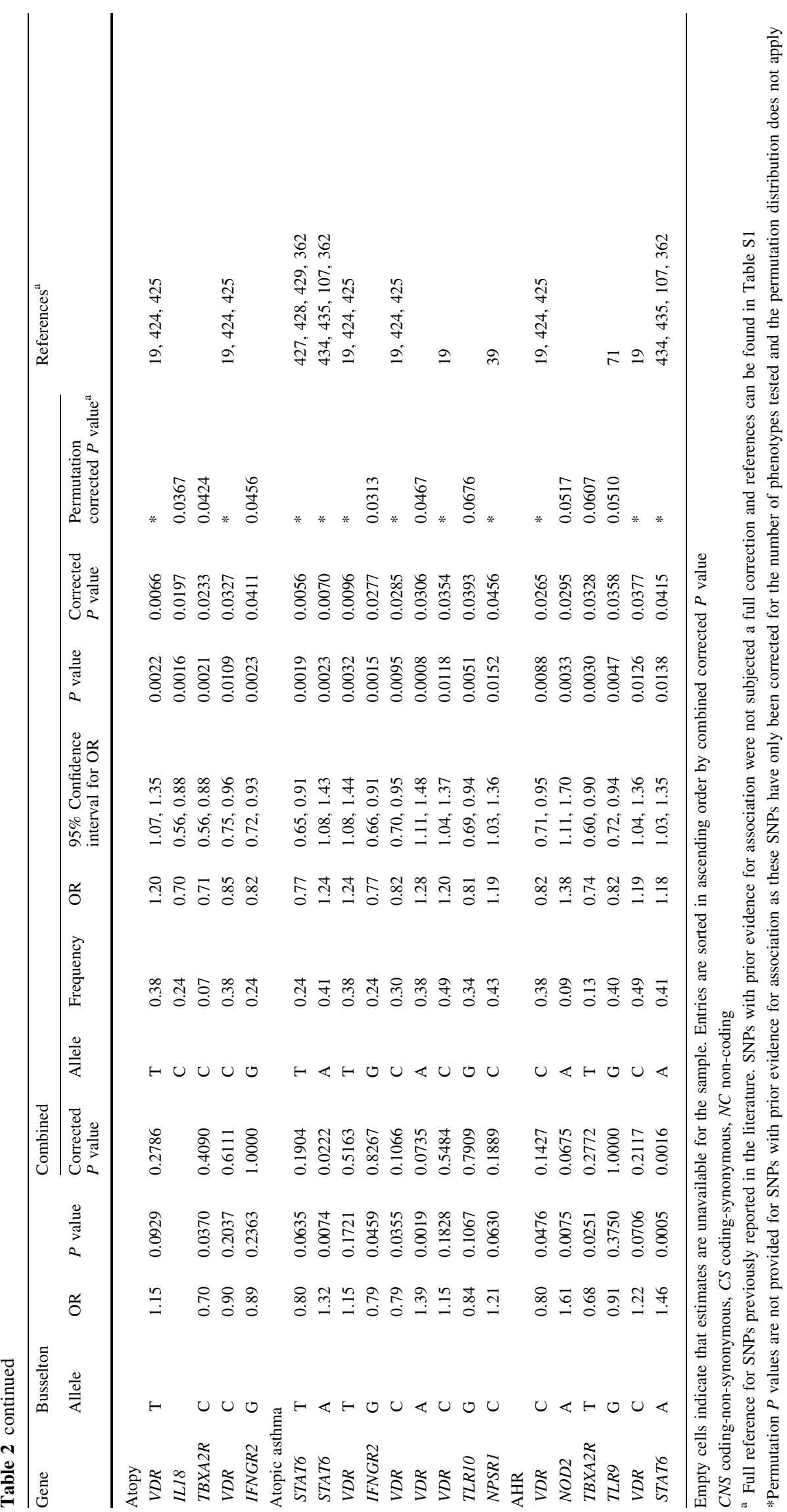


Table 3 Significant associations between asthma, atopy, atopic asthma, and AHR in the individual samples

\begin{tabular}{|c|c|c|c|c|c|c|c|c|c|}
\hline \multicolumn{4}{|c|}{ Sample } & \multicolumn{3}{|l|}{ CAPPS } & \multicolumn{3}{|l|}{ SAGE } \\
\hline Gene & SNP & Chromosome & Function & Allele Frequency OR & $\begin{array}{l}P \\
\text { value }\end{array}$ & $\begin{array}{l}\text { Corrected } \\
P \text { value }\end{array}$ & Allele Frequency OR & $\begin{array}{l}P \\
\text { value }\end{array}$ & $\begin{array}{l}\text { Corrected } \\
P \text { value }\end{array}$ \\
\hline
\end{tabular}

\begin{tabular}{|c|c|c|c|c|c|c|c|c|c|c|c|c|c|}
\hline \multicolumn{14}{|l|}{ Asthma } \\
\hline EDN1 & rs $1630736^{\mathrm{a}}$ & 6 & $\mathrm{NC}$ & A & 0.47 & 0.42 & 0.0028 & 0.0085 & A & 0.48 & 0.91 & 0.5869 & 1.0000 \\
\hline$V D R$ & $\mathrm{rs} 2853564^{\mathrm{a}}$ & 12 & $\mathrm{NC}$ & $\mathrm{G}$ & 0.38 & 2.46 & 0.0040 & 0.0120 & $\mathrm{G}$ & 0.36 & 1.15 & 0.4613 & 1.0000 \\
\hline IL10 & rs $3024492^{a}$ & 1 & $\mathrm{NC}$ & $\mathrm{T}$ & 0.22 & 2.06 & 0.0096 & 0.0289 & $\mathrm{~T}$ & 0.24 & 0.56 & 0.0048 & 0.0145 \\
\hline$I L 1 B$ & $\mathrm{rs} 16944^{\mathrm{a}}$ & 2 & $\mathrm{NC}$ & $\mathrm{T}$ & 0.37 & 2.13 & 0.0121 & 0.0364 & $\mathrm{~T}$ & 0.37 & 0.97 & 0.8539 & 1.0000 \\
\hline$C D 14$ & rs 4914 & 5 & $\mathrm{CS}$ & $\mathrm{C}$ & 0.11 & 1.25 & 0.6370 & 1.0000 & $\mathrm{C}$ & 0.10 & 0.36 & 0.0029 & 0.0260 \\
\hline IL10 & rs3024498 & 1 & $\mathrm{NC}$ & $\mathrm{G}$ & 0.23 & 2.06 & 0.0096 & 0.1735 & G & 0.24 & 0.55 & 0.0028 & 0.0498 \\
\hline$V D R$ & rs $1540339^{a}$ & 12 & $\mathrm{NC}$ & $\mathrm{T}$ & 0.40 & 1.00 & 1.0000 & 1.0000 & $\mathrm{~T}$ & 0.36 & 1.13 & 0.5210 & 1.0000 \\
\hline NPSR1 & rs $2609234^{\mathrm{a}}$ & 7 & $\mathrm{NC}$ & A & 0.17 & 1.27 & 0.4922 & 1.0000 & $\mathrm{~A}$ & 0.16 & 1.23 & 0.3646 & 1.0000 \\
\hline$V D R$ & rs $2239186^{a}$ & 12 & $\mathrm{NC}$ & G & 0.24 & 1.47 & 0.1879 & 0.5637 & $\mathrm{G}$ & 0.22 & 0.90 & 0.6505 & 1.0000 \\
\hline C5 & rs7033790 & 9 & $\mathrm{NC}$ & $\mathrm{T}$ & 0.18 & 0.80 & 0.5045 & 1.0000 & $\mathrm{~T}$ & 0.21 & 1.19 & 0.4307 & 1.0000 \\
\hline C5 & rs2416810 & 9 & $\mathrm{NC}$ & $\mathrm{T}$ & 0.19 & 0.94 & 0.8575 & 1.0000 & $\mathrm{~T}$ & 0.19 & 0.80 & 0.3107 & 1.0000 \\
\hline EDN1 & rs9369217 & 6 & $\mathrm{NC}$ & A & 0.15 & 1.88 & 0.1412 & 1.0000 & A & 0.14 & 1.10 & 0.7054 & 1.0000 \\
\hline CCL5 & rs4795095 & 17 & $\mathrm{NC}$ & $\mathrm{G}$ & 0.14 & 1.07 & 0.8527 & 1.0000 & $\mathrm{G}$ & 0.11 & 0.64 & 0.1806 & 1.0000 \\
\hline CCL5 & $\mathrm{rs} 2107538^{\mathrm{a}}$ & 17 & $\mathrm{NC}$ & A & 0.21 & 1.17 & 0.6308 & 1.0000 & A & 0.19 & 0.89 & 0.6325 & 1.0000 \\
\hline \multicolumn{14}{|l|}{ Atopy } \\
\hline IL18 & rs $360717^{\mathrm{a}}$ & 11 & $\mathrm{NC}$ & A & 0.25 & 1.91 & 0.0024 & 0.0073 & A & 0.26 & 1.08 & 0.6669 & 1.0000 \\
\hline IL18 & rs549908 & 11 & $\mathrm{CS}$ & G & 0.28 & 1.94 & 0.0015 & 0.0180 & $\mathrm{G}$ & 0.29 & 1.09 & 0.6095 & 1.0000 \\
\hline$I R F 2$ & rs1124191 & 4 & $\mathrm{NC}$ & A & 0.23 & 0.44 & 0.0004 & 0.0246 & A & 0.26 & 1.06 & 0.7297 & 1.0000 \\
\hline EDN1 & rs $1630736^{\mathrm{a}}$ & 6 & $\mathrm{NC}$ & A & 0.47 & 0.63 & 0.0082 & 0.0246 & $\mathrm{~A}$ & 0.48 & 0.85 & 0.3023 & 0.9069 \\
\hline$L T C 4 S$ & rs166624 & 5 & $\mathrm{NC}$ & $\mathrm{T}$ & 0.16 & 0.47 & 0.0028 & 0.0252 & $\mathrm{~T}$ & 0.14 & 1.03 & 0.9104 & 1.0000 \\
\hline IL18 & rs360712 & 11 & $\mathrm{CS}$ & $\mathrm{C}$ & 0.24 & 1.93 & 0.0026 & 0.0312 & $\mathrm{C}$ & 0.25 & 1.06 & 0.7277 & 1.0000 \\
\hline$C C L 2$ & $\mathrm{rs} 1024611^{\mathrm{a}}$ & 17 & $\mathrm{NC}$ & $\mathrm{C}$ & 0.32 & 1.69 & 0.0107 & 0.0321 & $\mathrm{C}$ & 0.32 & 1.05 & 0.7932 & 1.0000 \\
\hline CCL11 & rs $17809012^{a}$ & 17 & $\mathrm{NC}$ & G & 0.43 & 0.64 & 0.0123 & 0.0367 & $\mathrm{G}$ & 0.45 & 0.95 & 0.7131 & 1.0000 \\
\hline$I L 1 B$ & rs1143627 & 2 & $\mathrm{NC}$ & $\mathrm{G}$ & 0.37 & 1.67 & 0.0065 & 0.0393 & $\mathrm{G}$ & 0.38 & 1.02 & 0.8774 & 1.0000 \\
\hline$I L 1 B$ & $\mathrm{rs} 16944^{\mathrm{a}}$ & 2 & $\mathrm{NC}$ & $\mathrm{T}$ & 0.37 & 1.59 & 0.0144 & 0.0434 & $\mathrm{~T}$ & 0.37 & 1.05 & 0.7562 & 1.0000 \\
\hline CTLA4 & rs $5742909^{a}$ & 2 & $\mathrm{NC}$ & $\mathrm{A}$ & 0.08 & 0.44 & 0.0150 & 0.0449 & $\mathrm{~A}$ & 0.09 & 1.20 & 0.4999 & 1.0000 \\
\hline$C 5$ & rs10985112 & 9 & $\mathrm{NC}$ & A & 0.08 & 1.06 & 0.8694 & 1.0000 & & & & & \\
\hline$V D R$ & $\mathrm{rs} 1540339^{\mathrm{a}}$ & 12 & $\mathrm{NC}$ & $\mathrm{T}$ & 0.40 & 0.95 & 0.7797 & 1.0000 & $\mathrm{~T}$ & 0.36 & 1.30 & 0.0995 & 0.2986 \\
\hline$V D R$ & rs $2853564^{\mathrm{a}}$ & 12 & $\mathrm{NC}$ & $\mathrm{G}$ & 0.38 & 1.16 & 0.4412 & 1.0000 & G & 0.36 & 1.09 & 0.5787 & 1.0000 \\
\hline NPSR1 & rs $2609234^{\mathrm{a}}$ & 7 & $\mathrm{NC}$ & A & 0.17 & 1.27 & 0.3045 & 0.9135 & A & 0.16 & 1.14 & 0.4944 & 1.0000 \\
\hline C5 & rs2159776 & 9 & $\mathrm{NC}$ & G & 0.44 & 0.77 & 0.1469 & 1.0000 & G & 0.46 & 0.90 & 0.4886 & 1.0000 \\
\hline C5 & $\mathrm{rs} 17611^{\mathrm{a}}$ & 9 & CNS & $\mathrm{G}$ & 0.53 & 0.76 & 0.1166 & 0.3498 & A & 0.43 & 0.95 & 0.7146 & 1.0000 \\
\hline$F L G$ & rs12730241 & 1 & $\mathrm{NC}$ & & & & & & & & & & \\
\hline PTGDR & rs803022 & 14 & $\mathrm{NC}$ & $\mathrm{T}$ & 0.19 & 0.83 & 0.4247 & 1.0000 & $\mathrm{~T}$ & 0.21 & 1.36 & 0.1059 & 1.0000 \\
\hline$A L O X 5$ & rs892691 & 10 & $\mathrm{NC}$ & $\mathrm{T}$ & 0.28 & 1.00 & 1.0000 & 1.0000 & $\mathrm{~T}$ & 0.25 & 1.03 & 0.8575 & 1.0000 \\
\hline \multicolumn{14}{|c|}{ Atopic Asthma } \\
\hline IFNGR2 & rs2834213 & 21 & $\mathrm{NC}$ & $\mathrm{G}$ & 0.22 & 0.24 & 0.0004 & 0.0073 & $\mathrm{G}$ & 0.24 & 1.16 & 0.5860 & 1.0000 \\
\hline EDN1 & rs $1630736^{\mathrm{a}}$ & 6 & $\mathrm{NC}$ & A & 0.47 & 0.37 & 0.0044 & 0.0132 & A & 0.48 & 0.85 & 0.4911 & 1.0000 \\
\hline IL10 & rs $3024492^{\mathrm{a}}$ & 1 & $\mathrm{NC}$ & $\mathrm{T}$ & 0.22 & 2.46 & 0.0084 & 0.0251 & $\mathrm{~T}$ & 0.24 & 0.47 & 0.0034 & 0.0101 \\
\hline TLR10 & rs10776483 & 4 & $\mathrm{CS}$ & $\mathrm{C}$ & 0.21 & 0.60 & 0.1551 & 1.0000 & $\mathrm{C}$ & 0.22 & 0.41 & 0.0014 & 0.0110 \\
\hline TLR10 & rs11096957 & 4 & $\mathrm{CNS}$ & $\mathrm{G}$ & 0.36 & 0.71 & 0.2731 & 1.0000 & $\mathrm{G}$ & 0.38 & 0.48 & 0.0023 & 0.0179 \\
\hline IL5 & rs 743562 & 5 & $\mathrm{NC}$ & $\mathrm{T}$ & 0.41 & 0.95 & 0.8728 & 1.0000 & $\mathrm{~T}$ & 0.43 & 1.79 & 0.0053 & 0.0392 \\
\hline PHF11 & rs $2247119^{a}$ & 13 & $\mathrm{NC}$ & $\mathrm{C}$ & 0.31 & 1.36 & 0.3832 & 1.0000 & $\mathrm{C}$ & 0.30 & 1.78 & 0.0146 & 0.0439 \\
\hline$V D R$ & rs $1540339^{a}$ & 12 & $\mathrm{NC}$ & $\mathrm{T}$ & 0.40 & 1.10 & 0.7630 & 1.0000 & $\mathrm{~T}$ & 0.36 & 1.27 & 0.2981 & 0.8943 \\
\hline$V D R$ & $\mathrm{rs} 2853564^{\mathrm{a}}$ & 12 & $\mathrm{NC}$ & G & 0.38 & 2.00 & 0.0532 & 0.1596 & $\mathrm{G}$ & 0.36 & 1.29 & 0.2569 & 0.7707 \\
\hline
\end{tabular}


Table 3 continued

\begin{tabular}{|c|c|c|c|c|c|c|c|c|c|c|c|c|c|}
\hline \multicolumn{4}{|l|}{ Sample } & \multicolumn{5}{|c|}{ CAPPS } & \multicolumn{5}{|l|}{ SAGE } \\
\hline Gene & SNP & Chromosome & Function & Allele & Frequency & OR & $\begin{array}{l}P \\
\text { value }\end{array}$ & $\begin{array}{l}\text { Corrected } \\
P \text { value }\end{array}$ & Allele & Frequency & OR & $\begin{array}{l}P \\
\text { value }\end{array}$ & $\begin{array}{l}\text { Corrected } \\
P \text { value }\end{array}$ \\
\hline STAT6 & $\operatorname{rs} 324011^{\mathrm{a}}$ & 12 & $\mathrm{NC}$ & A & 0.39 & 1.17 & 0.6308 & 1.0000 & A & 0.43 & 1.13 & 0.5875 & 1.0000 \\
\hline C5 & rs2159776 & 9 & $\mathrm{NC}$ & G & 0.44 & 0.65 & 0.1683 & 1.0000 & $\mathrm{G}$ & 0.46 & 0.93 & 0.7357 & 1.0000 \\
\hline ALOX5 & rs892691 & 10 & $\mathrm{NC}$ & $\mathrm{T}$ & 0.28 & 0.65 & 0.2550 & 1.0000 & $\mathrm{~T}$ & 0.25 & 1.43 & 0.2064 & 1.0000 \\
\hline SERPINA3 & rs4900239 & 14 & $\mathrm{NC}$ & $\mathrm{T}$ & 0.08 & 1.00 & 1.0000 & 1.0000 & $\mathrm{~T}$ & 0.09 & 1.00 & 1.0000 & 1.0000 \\
\hline$C 5$ & $\mathrm{rs} 17611^{\mathrm{a}}$ & 9 & $\mathrm{CNS}$ & $\mathrm{G}$ & 0.53 & 0.62 & 0.1070 & 0.3210 & A & 0.43 & 0.91 & 0.6732 & 1.0000 \\
\hline$P T G D R$ & rs803022 & 14 & $\mathrm{NC}$ & $\mathrm{T}$ & 0.19 & 1.50 & 0.3157 & 1.0000 & $\mathrm{~T}$ & 0.21 & 1.48 & 0.1439 & 1.0000 \\
\hline \multicolumn{14}{|l|}{ AHR } \\
\hline$P G D S$ & rs 8336 & 4 & $\mathrm{NC}$ & A & 0.40 & 1.25 & 0.1661 & 1.0000 & G & 0.59 & 0.52 & 0.0007 & 0.0052 \\
\hline$H N M T$ & rs1349992 & 2 & $\mathrm{NC}$ & $\mathrm{A}$ & 0.45 & 1.08 & 0.6715 & 1.0000 & A & 0.39 & 1.94 & 0.0018 & 0.0216 \\
\hline$P G D S$ & rs2171381 & 4 & $\mathrm{NC}$ & $\mathrm{T}$ & 0.44 & 0.97 & 0.8711 & 1.0000 & $\mathrm{~T}$ & 0.43 & 0.54 & 0.0036 & 0.0278 \\
\hline$C 5$ & rs 10985112 & 9 & $\mathrm{NC}$ & $\mathrm{A}$ & 0.08 & 1.44 & 0.2265 & 1.0000 & & & & & \\
\hline STAT6 & $\mathrm{rs} 324011^{\mathrm{a}}$ & 12 & $\mathrm{NC}$ & $\mathrm{A}$ & 0.39 & 1.05 & 0.7533 & 1.0000 & A & 0.43 & 0.96 & 0.8501 & 1.0000 \\
\hline STAT6 & rs167769 & 12 & $\mathrm{NC}$ & $\mathrm{T}$ & 0.38 & 0.96 & 0.8119 & 1.0000 & $\mathrm{~T}$ & 0.42 & 0.84 & 0.3487 & 1.0000 \\
\hline$H N M T$ & rs6755148 & 2 & $\mathrm{NC}$ & $\mathrm{C}$ & 0.27 & 1.00 & 1.0000 & 1.0000 & $\mathrm{C}$ & 0.30 & 0.70 & 0.0908 & 1.0000 \\
\hline$V D R$ & rs $3782905^{\mathrm{a}}$ & 12 & $\mathrm{NC}$ & $\mathrm{C}$ & 0.28 & 1.16 & 0.4206 & 1.0000 & $\mathrm{C}$ & 0.31 & 0.94 & 0.7557 & 1.0000 \\
\hline \multirow[t]{2}{*}{ Gene } & \multicolumn{3}{|l|}{ SLSJ } & \multicolumn{5}{|c|}{ Busselton } & \multicolumn{5}{|c|}{ Combined } \\
\hline & Allele Frequ & $\begin{array}{ll}\text { ncy } & P \\
& \text { valu }\end{array}$ & $\begin{array}{l}\text { Correc } \\
P \text { valu }\end{array}$ & & lele Frequen & ncy $\mathrm{OF}$ & $\begin{array}{ll}\mathrm{R} & P \\
& \text { value }\end{array}$ & $\begin{array}{l}\text { Corrected } \\
P \text { value }\end{array}$ & d Allele & Frequency & OR & $\begin{array}{l}P \\
\text { value }\end{array}$ & $\begin{array}{l}\text { Corrected } \\
P \text { value }\end{array}$ \\
\hline
\end{tabular}

Asthma

\begin{tabular}{|c|c|c|c|c|c|c|c|c|c|c|c|c|c|c|}
\hline EDN1 & A & 0.50 & 1.02 & 0.8307 & 1.0000 & A & 0.44 & 1.00 & 0.9491 & 1.0000 & A & 0.46 & 0.960 .4772 & 1.0000 \\
\hline$V D R$ & $\mathrm{G}$ & 0.38 & 0.79 & 0.0315 & 0.0944 & $\mathrm{G}$ & 0.42 & 0.96 & 0.6108 & 1.0000 & $\mathrm{G}$ & 0.39 & 0.960 .4606 & 1.0000 \\
\hline ILIO & $\mathrm{T}$ & 0.24 & 0.95 & 0.6683 & 1.0000 & $\mathrm{~T}$ & 0.29 & 1.11 & 0.2063 & 0.6189 & $\mathrm{~T}$ & 0.26 & $1.03 \quad 0.6519$ & 1.0000 \\
\hline$I L 1 B$ & $\mathrm{~T}$ & 0.27 & 1.11 & 0.3850 & 1.0000 & $\mathrm{~T}$ & 0.35 & 1.04 & 0.6558 & 1.0000 & $\mathrm{~T}$ & 0.35 & $\begin{array}{ll}1.08 & 0.2158\end{array}$ & 0.6474 \\
\hline$C D 14$ & $\mathrm{C}$ & 0.11 & 0.84 & 0.3063 & 1.0000 & $\mathrm{C}$ & 0.12 & 1.14 & 0.2672 & 1.0000 & $\mathrm{C}$ & 0.11 & 0.960 .6742 & 1.0000 \\
\hline ILIO & $\mathrm{G}$ & 0.24 & 0.95 & 0.6683 & 1.0000 & G & 0.29 & 1.12 & 0.1815 & 1.0000 & G & 0.26 & $\begin{array}{ll}1.03 & 0.6577\end{array}$ & 1.0000 \\
\hline$V D R$ & $\mathrm{~T}$ & 0.41 & 1.48 & 0.0006 & 0.0017 & $\mathrm{~T}$ & 0.37 & 1.07 & 0.4189 & 1.0000 & $\mathrm{~T}$ & 0.38 & $\begin{array}{ll}1.17 & 0.0089\end{array}$ & 0.0267 \\
\hline NPSR1 & A & 0.19 & 0.69 & 0.0075 & 0.0224 & A & 0.16 & 1.15 & 0.1710 & 0.5130 & A & 0.16 & $1.00 \quad 0.9767$ & 1.0000 \\
\hline$V D R$ & G & 0.20 & 1.45 & 0.0093 & 0.0280 & G & 0.21 & 1.09 & 0.3605 & 1.0000 & $\mathrm{G}$ & 0.22 & 1.170 .0280 & 0.0841 \\
\hline$C 5$ & $\mathrm{~T}$ & 0.24 & 0.68 & 0.0016 & 0.0304 & $\mathrm{~T}$ & 0.23 & 1.15 & 0.1306 & 1.0000 & $\mathrm{~T}$ & 0.22 & 0.960 .5788 & 1.0000 \\
\hline$C 5$ & $\mathrm{~T}$ & 0.17 & 1.51 & 0.0026 & 0.0478 & $\mathrm{~T}$ & 0.19 & 0.98 & 0.8714 & 1.0000 & $\mathrm{~T}$ & 0.19 & $\begin{array}{ll}1.09 & 0.2470\end{array}$ & 1.0000 \\
\hline EDN1 & A & 0.13 & 0.99 & 0.9356 & 1.0000 & A & 0.18 & 1.37 & 0.0013 & 0.0160 & A & 0.16 & 1.260 .0036 & 0.0435 \\
\hline CCL5 & G & 0.10 & 1.40 & 0.0422 & 0.2054 & G & 0.10 & 0.71 & 0.0073 & 0.0370 & G & 0.11 & $0.90 \quad 0.2571$ & 1.0000 \\
\hline CCL5 & A & 0.18 & 1.06 & 0.6436 & 1.0000 & $\mathrm{~A}$ & 0.19 & 0.79 & 0.0144 & 0.0433 & A & 0.19 & 0.890 .1086 & 0.3258 \\
\hline \multicolumn{15}{|l|}{ Atopy } \\
\hline IL18 & A & 0.27 & 0.96 & 0.7630 & 1.0000 & & & & & & A & 0.26 & 1.120 .1958 & 0.5874 \\
\hline ILI8 & G & 0.30 & 1.05 & 0.6856 & 1.0000 & G & 0.31 & 1.08 & 0.3958 & 1.0000 & G & 0.30 & 1.130 .0503 & 0.6035 \\
\hline$I R F 2$ & A & 0.25 & 0.88 & 0.3047 & 1.0000 & A & 0.24 & 1.00 & 0.9816 & 1.0000 & A & 0.24 & 0.910 .1442 & 1.0000 \\
\hline EDN1 & G & 0.50 & 0.88 & 0.2216 & 0.6648 & A & 0.44 & 0.89 & 0.1636 & 0.4908 & A & 0.46 & 0.920 .1255 & 0.3765 \\
\hline$L T C 4 S$ & $\mathrm{~T}$ & 0.16 & 1.12 & 0.4397 & 1.0000 & $\mathrm{~T}$ & 0.16 & 1.04 & 0.7236 & 1.0000 & $\mathrm{~T}$ & 0.15 & $0.98 \quad 0.8261$ & 1.0000 \\
\hline IL18 & $\mathrm{C}$ & 0.24 & 0.91 & 0.4838 & 1.0000 & $\mathrm{C}$ & 0.26 & 1.13 & 0.1805 & 1.0000 & $\mathrm{C}$ & 0.25 & 1.110 .1026 & 1.0000 \\
\hline$C C L 2$ & $\mathrm{C}$ & 0.32 & 1.09 & 0.4393 & 1.0000 & $\mathrm{C}$ & 0.27 & 1.03 & 0.7264 & 1.0000 & $\mathrm{C}$ & 0.30 & 1.100 .1202 & 0.3606 \\
\hline CCL11 & G & 0.33 & 0.96 & 0.7148 & 1.0000 & G & 0.44 & 1.03 & 0.7047 & 1.0000 & G & 0.43 & $0.95 \quad 0.3872$ & 1.0000 \\
\hline$I L 1 B$ & G & 0.27 & 1.16 & 0.2405 & 1.0000 & G & 0.34 & 1.05 & 0.5861 & 1.0000 & G & 0.35 & 1.120 .0573 & 0.3436 \\
\hline$I L 1 B$ & $\mathrm{~T}$ & 0.27 & 1.16 & 0.2405 & 0.7215 & $\mathrm{~T}$ & 0.35 & 1.06 & 0.5064 & 1.0000 & $\mathrm{~T}$ & 0.35 & 1.130 .0503 & 0.1508 \\
\hline CTLA4 & A & 0.11 & 0.82 & 0.2552 & 0.7656 & A & 0.09 & 0.82 & 0.1676 & 0.5028 & A & 0.09 & 0.820 .0390 & 0.1169 \\
\hline
\end{tabular}


Table 3 continued

\begin{tabular}{|c|c|c|c|c|c|c|c|c|c|c|c|c|c|c|c|}
\hline \multirow[t]{2}{*}{ Gene } & \multicolumn{5}{|l|}{ SLSJ } & \multicolumn{5}{|c|}{ Busselton } & \multicolumn{5}{|c|}{ Combined } \\
\hline & Allele & Frequency & OR & $\begin{array}{l}P \\
\text { value }\end{array}$ & $\begin{array}{l}\text { Corrected } \\
P \text { value }\end{array}$ & Allele & Frequency & OR & $\begin{array}{l}P \\
\text { value }\end{array}$ & $\begin{array}{l}\text { Corrected } \\
P \text { value }\end{array}$ & Allele & Frequency & OR & $\begin{array}{l}P \\
\text { value }\end{array}$ & $\begin{array}{l}\text { Corrected } \\
P \text { value }\end{array}$ \\
\hline C5 & A & 0.07 & 2.08 & 0.0007 & 0.0136 & A & 0.08 & 0.76 & 0.0709 & 1.0000 & A & 0.08 & 1.07 & 0.5638 & 1.0000 \\
\hline$V D R$ & $\mathrm{~T}$ & 0.41 & 1.35 & 0.0090 & 0.0270 & $\mathrm{~T}$ & 0.37 & 1.15 & 0.0929 & 0.2786 & $\mathrm{~T}$ & 0.38 & 1.20 & 0.0022 & 0.0066 \\
\hline$V D R$ & G & 0.38 & 0.75 & 0.0099 & 0.0296 & G & 0.42 & 0.99 & 0.8637 & 1.0000 & G & 0.39 & 0.94 & 0.3037 & 0.9111 \\
\hline NPSR1 & A & 0.19 & 0.77 & 0.0583 & 0.1750 & A & 0.16 & 0.74 & 0.0065 & 0.0194 & A & 0.16 & 0.84 & 0.0247 & 0.0742 \\
\hline$C 5$ & G & 0.51 & 0.81 & 0.0463 & 0.8637 & G & 0.46 & 1.30 & 0.0014 & 0.0253 & G & 0.47 & 1.03 & 0.6496 & 1.0000 \\
\hline C5 & A & 0.39 & 1.08 & 0.4653 & 1.0000 & A & 0.44 & 0.81 & 0.0108 & 0.0325 & A & 0.44 & 0.95 & 0.3298 & 0.9894 \\
\hline$F L G$ & & & & & & A & 0.15 & 0.71 & 0.0029 & 0.0346 & A & 0.15 & 0.71 & 0.0029 & 0.0346 \\
\hline PTGDR & $\mathrm{T}$ & 0.21 & 1.06 & 0.6830 & 1.0000 & $\mathrm{~T}$ & 0.20 & 0.73 & 0.0016 & 0.0353 & $\mathrm{~T}$ & 0.20 & 0.89 & 0.1022 & 1.0000 \\
\hline$A L O X 5$ & $\mathrm{~T}$ & 0.30 & 1.07 & 0.5687 & 1.0000 & $\mathrm{~T}$ & 0.27 & 0.75 & 0.0020 & 0.0363 & $\mathrm{~T}$ & 0.27 & 0.89 & 0.0791 & 1.0000 \\
\hline \multicolumn{16}{|c|}{ Atopic asthma } \\
\hline IFNGR2 & G & 0.28 & 0.78 & 0.0568 & 1.0000 & G & 0.24 & 0.79 & 0.0459 & 0.8267 & G & 0.24 & 0.77 & 0.0015 & 0.0277 \\
\hline EDN1 & G & 0.50 & 0.93 & 0.5528 & 1.0000 & A & 0.44 & 0.89 & 0.2725 & 0.8175 & A & 0.46 & 0.92 & 0.2170 & 0.6510 \\
\hline IL10 & $\mathrm{T}$ & 0.24 & 1.03 & 0.8401 & 1.0000 & $\mathrm{~T}$ & 0.29 & 1.07 & 0.5342 & 1.0000 & $\mathrm{~T}$ & 0.26 & 1.02 & 0.7814 & 1.0000 \\
\hline TLR10 & $\mathrm{C}$ & 0.17 & 0.98 & 0.8802 & 1.0000 & $\mathrm{C}$ & 0.19 & 0.84 & 0.1788 & 1.0000 & $\mathrm{C}$ & 0.20 & 0.80 & 0.0146 & 0.1124 \\
\hline TLR10 & G & 0.29 & 0.91 & 0.4784 & 1.0000 & G & 0.32 & 0.84 & 0.1067 & 0.7909 & G & 0.34 & 0.81 & 0.0051 & 0.0393 \\
\hline IL5 & $\mathrm{T}$ & 0.44 & 0.82 & 0.0940 & 0.6795 & $\mathrm{~T}$ & 0.42 & 0.91 & 0.3607 & 1.0000 & $\mathrm{~T}$ & 0.42 & 0.95 & 0.4660 & 1.0000 \\
\hline PHF11 & $\mathrm{C}$ & 0.27 & 0.81 & 0.1161 & 0.3483 & $\mathrm{C}$ & 0.30 & 1.02 & 0.8287 & 1.0000 & $\mathrm{C}$ & 0.30 & 1.02 & 0.7862 & 1.0000 \\
\hline$V D R$ & $\mathrm{~T}$ & 0.41 & 1.41 & 0.0068 & 0.0203 & $\mathrm{~T}$ & 0.37 & 1.15 & 0.1721 & 0.5163 & $\mathrm{~T}$ & 0.38 & 1.24 & 0.0032 & 0.0096 \\
\hline$V D R$ & G & 0.38 & 0.73 & 0.0110 & 0.0331 & G & 0.42 & 0.95 & 0.6355 & 1.0000 & G & 0.39 & 0.93 & 0.2863 & 0.8589 \\
\hline STAT6 & A & 0.38 & 1.20 & 0.1354 & 0.4062 & A & 0.42 & 1.32 & 0.0074 & 0.0222 & A & 0.41 & 1.24 & 0.0023 & 0.0070 \\
\hline C5 & G & 0.51 & 0.78 & 0.0337 & 0.6281 & G & 0.46 & 1.38 & 0.0015 & 0.0272 & G & 0.47 & 1.03 & 0.6315 & 1.0000 \\
\hline$A L O X 5$ & $\mathrm{~T}$ & 0.30 & 1.03 & 0.8011 & 1.0000 & $\mathrm{~T}$ & 0.27 & 0.70 & 0.0016 & 0.0283 & $\mathrm{~T}$ & 0.27 & 0.86 & 0.0546 & 0.9835 \\
\hline SERPINA3 & $\mathrm{T}$ & 0.12 & 1.12 & 0.5277 & 1.0000 & $\mathrm{~T}$ & 0.11 & 0.61 & 0.0037 & 0.0326 & $\mathrm{~T}$ & 0.10 & 0.84 & 0.1251 & 1.0000 \\
\hline C5 & A & 0.39 & 1.12 & 0.3140 & 0.9420 & A & 0.44 & 0.77 & 0.0120 & 0.0360 & A & 0.44 & 0.94 & 0.4039 & 1.0000 \\
\hline PTGDR & $\mathrm{T}$ & 0.21 & 1.03 & 0.8273 & 1.0000 & $\mathrm{~T}$ & 0.20 & 0.67 & 0.0021 & 0.0457 & $\mathrm{~T}$ & 0.20 & 0.89 & 0.1985 & 1.0000 \\
\hline \multicolumn{16}{|l|}{ AHR } \\
\hline$P G D S$ & A & 0.41 & 0.86 & 0.2318 & 1.0000 & A & 0.40 & 0.84 & 0.1285 & 0.9943 & A & 0.41 & 1.02 & 0.8173 & 1.0000 \\
\hline$H N M T$ & A & 0.39 & 1.10 & 0.4459 & 1.0000 & A & 0.44 & 0.83 & 0.0876 & 1.0000 & A & 0.42 & 1.04 & 0.5786 & 1.0000 \\
\hline$P G D S$ & $\mathrm{~T}$ & 0.43 & 1.03 & 0.8033 & 1.0000 & $\mathrm{~T}$ & 0.40 & 0.95 & 0.6293 & 1.0000 & $\mathrm{~T}$ & 0.42 & 0.92 & 0.2279 & 1.0000 \\
\hline C5 & A & 0.07 & 1.95 & 0.0022 & 0.0413 & A & 0.08 & 0.89 & 0.5794 & 1.0000 & A & 0.08 & 1.31 & 0.0379 & 0.7107 \\
\hline STAT6 & A & 0.38 & 1.06 & 0.6611 & 1.0000 & A & 0.42 & 1.46 & 0.0005 & 0.0016 & A & 0.41 & 1.18 & 0.0138 & 0.0415 \\
\hline STAT6 & $\mathrm{T}$ & 0.36 & 1.09 & 0.5219 & 1.0000 & $\mathrm{~T}$ & 0.40 & 1.53 & 0.0001 & 0.0019 & $\mathrm{~T}$ & 0.39 & 1.17 & 0.0205 & 0.3686 \\
\hline$H N M T$ & $\mathrm{C}$ & 0.23 & 1.11 & 0.5034 & 1.0000 & $\mathrm{C}$ & 0.28 & 1.42 & 0.0026 & 0.0316 & $\mathrm{C}$ & 0.28 & 1.14 & 0.0933 & 1.0000 \\
\hline$V D R$ & $\mathrm{C}$ & 0.29 & 0.83 & 0.1608 & 0.4824 & $\mathrm{C}$ & 0.31 & 0.74 & 0.0131 & 0.0392 & $\mathrm{C}$ & 0.30 & 0.85 & 0.0352 & 0.1057 \\
\hline
\end{tabular}

Empty cells indicate that estimates are unavailable for the sample. Entries have been sorted by corrected $P$ value starting with the CAPPS cohort, then SAGE, SLSJ and Busselton

CNS coding-non-synonymous, CS coding synonymous $N C$ non-coding

${ }^{a}$ Indicates SNPs with prior evidence for association (these SNPs have only been corrected for the number of phenotypes tested)

remaining families. Four sets of twins were identified in the CAPPS cohort (2 monozygotic and 2 dizygotic), the dizygotic twins were retained; a single sib was chosen from the monozygotic pairs for inclusion in the analysis. In the case-control sample of Busselton, we identified 73 parent-offspring relationships and 52 sib pairs. To address these relationships we eliminated 125 samples. We moreover identified two samples that were likely to be of Asian descent, inclusion of which (in a case-control design) could contribute to spurious results because of population differences in allele frequency. We also identified two duplicate samples (identical at all loci) but as the two samples differed in phenotype (one case and one control) both samples were removed. Removal of these 
samples resulted in a total of 644 asthma cases and 751 controls available for analysis.

\section{Combined results}

We examined the evidence for association with 93 candidate genes by using direct and indirect tests of association in a joint analysis that utilized all samples and also with individual panels to evaluate both heterogeneity and individual-panel contributions to the joint analysis. Detailed results are provided for the 26 SNPs which showed trends for association in the combined analysis, after allowing for the number of SNPs within their respective genes (Table 2). Results for all panels, genes, and SNPs by phenotype are available in the Supplement (Supplementary Table S3). $P$ values have been adjusted for both the effective number of independent SNPs tested in a gene as well as the effective number of independent phenotypes tested, which was estimated to be 3 (see "Methods"), and confirmed by permutation tests where deemed significant. None of the results resist a correction for the total number of genes tested using a Bonferroni correction.

Because this set of genes has been associated with asthma or asthma-related traits, a global examination of our evidence for and against association that includes all genes and SNPs may be more informative than an examination of individual associations. When quantile-quantile plots for the $\chi^{2}$ test statistics are compared to the expected distribution, we observe a deviation in the shoulder of the distribution, but overall no gross inflation in the tail of the test statistics (Fig. 2). These observations are consistent with a candidate gene study, and indicate an excess of SNPs with modest evidence for association. These results are both consistent and to be expected from a set of candidate genes of relatively small effect size $\mathrm{OR}<1.4$, although the possibility remains that the inflation of the $\chi^{2}$ test statistic is the result of unresolved population stratification in the Busselton sample or artifacts such as nonrandom absence of genotype data. Our results indicate that even larger sample sizes than the current study $(N=5,565)$ are needed to clearly delineate the causal variants for these candidate genes.

The genes for which we found trends for replication with asthma and allergic phenotypes after correcting for the number of tests at each locus (see "Methods") are among those most consistently replicated in the literature; these include IL13, STAT6, and TBXA2R (Ober and Hoffjan 2006). ILI3 and VDR have been previously examined in the CAPPS (He et al. 2003) (IL13) and SLSJ (Poon et al. 2004) (VDR) panels. A sensitivity analysis was conducted by excluding the CAPPS cohort from the
Fig. 2 For each of the four phenotypes, a qq plot of the $\chi^{2}$ test statistics is constructed by ranking the values of the test statistic from smallest to largest (order statistic) and plotting them against the expected distribution. Values for each quantile are shown in black. The expected distribution is based upon 861 independent $\chi^{2}$ tests. Lines indicate the $95 \%$ confidence bands of the expected distribution

\section{QQ-plot of Combined Test Statistics Versus Chi-square ( $\mathrm{N}=861)$}
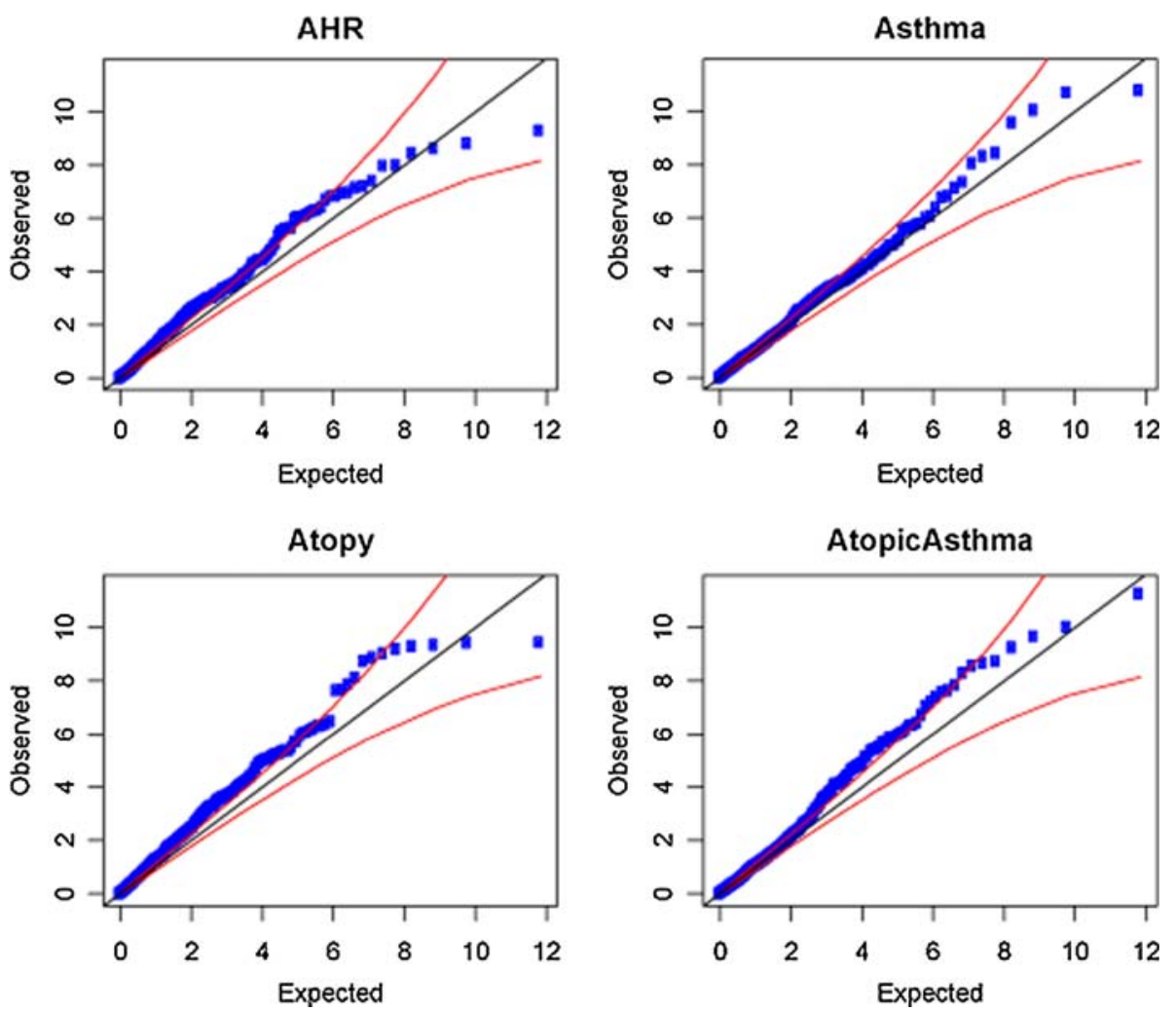
IL13 combined analysis with a small change in the adjusted $P$ value for asthma rs2243204 $(P=0.0126$ to $P=0.0328)$, rs20541 $(P=0.0342$ to $P=0.08016)$, and rs1295686 $(P=0.0424$ to $P=0.11406)$. For the $V D R$ analysis we excluded the 227 families recruited from the Saguenay-Lac-Saint-Jean region of Quebec as these families may have been used in the previous analysis. Families recruited from Quebec City $(N=79)$ were retained as they were not utilized in the prior study. The adjusted $P$ values are for rs1540339 (asthma $P=0.0267$ to $P=0.7377$, atopy $P=0.0066$ to $P=0.0894$, atopic asthma $P=0.0096$ to $P=0.1670$ ), rs731236 (atopy $P=0.0327 \quad$ to $P=0.2119$, AHR $P=0.0265$ to $P=0.05814$ ), rs2239185 (atopic asthma $P=0.0354$ to $P=0.4101$ AHR $P=0.0377$ to $P=0.15)$, rs3782905 (atopic asthma $P=0.0285$ to $P=0.1700$ and for rs11608702 (atopic asthma $P=0.0306$ to $P=0.0408$ ).

With related phenotypes, we expected to see concordance of the associations with more than one phenotype. IFNGR2, TBXA2R, STAT6, and VDR are all associated with more than one phenotype. Genes showing trends for association with one or more phenotypes were examined for nominal associations or trends with other phenotypes. The following genes have an unadjusted $P$ value $<0.05$ for the additional phenotype: TLR10 (asthma), TLR9 (asthma), and ILI3 (atopy and AHR). VDR demonstrates evidence for association with all four phenotypes; ILI3 and IFNGR2 with three; and TLR10,TBXA2R, and TLR9 with two. None of these candidate genes are in regions recently identified in the first genome-wide association study for asthma (Moffatt et al. 2007).

\section{Individual panel results}

We recognize that there may be important differences in phenotype expression, prevalence, gender distribution, and risk factors between childhood and adult asthma. Recent studies have suggested that there may be gender-specific responses to environmental exposures (Arshad and Hide 1992; Arshad et al. 2001; Gilliland et al. 2003; Li et al. 2000) and that the timing of environmental exposures during early childhood may be critically important (Melen et al. 2001; Salam et al. 2004). These differences have led researchers to speculate whether adult and childhood asthma are, in fact, the same disease (Martinez 2007).

In recognition of these potentially important differences, we present individual point estimates (OR and $P$ values) for each of the individual samples and the joint analysis. This allows researchers to evaluate the evidence for association in both childhood (CAPPS and SAGE) and adult samples (Table 3 and Supplementary Table S4), and facilitates future meta-analyses.
Caucasian only results

To further evaluate heterogeneity and sensitivity, we stratified the samples by ethnicity and examined the evidence for association in the Caucasian only sample (Supplementary Table S3). In general, the results were similar to the analysis where non-Caucasian samples were included in the combined analysis.

SNPs previously reported in the literature

We report on the associations for 154 SNPs previously reported in the literature that passed genotyping and entered the analysis (Supplementary Tables S1 and S4). About 104 of the 154 SNPs have been previously reported to be associated with an asthma related phenotype. For those 104 SNPs previously found to be associated in the literature we present the results correcting only for the number of independent phenotypes tested (direct test, Supplementary Table S4). For the 16 SNPs which showed trends for association in the combined analysis we present corrections using only the direct test, as well as correcting for both the number of SNPs and phenotypes tested in the analysis.

\section{Discussion}

The study was initiated at a time when the literature contained close to 100 published reports of association between genetic variants at candidate loci and asthma or atopy, and prior to the recent advent of genome-wide scans (Weidinger et al. 2008). The main message that is derived from the current study is that single alleles associated with asthma and atopy appear to have very modest effects (with ORs < 1.4) and that many published associations for asthma and atopy may be false-positives. The low rate of replication may also be due to small effect size, differences in phenotypic definition, differential environmental effects and/or genetic heterogeneity. A study such as this one provides a more realistic picture of genetic predisposition of asthma and atopy than hundreds of independent reports. Although we come to the conclusion that four studies with over 5,000 subjects is not enough to delineate false from true positive associations having very small effects, we believe that prioritization of loci to be validated can be enabled by reporting the combined and single-study results to the asthma and allergy community. We have also provided an extensive web resource (http://genapha. icapture.ubc.ca/) which includes access to online tools that interface our data with additional online resources, databases, and references. 
We accepted evidence for replication if any single SNP in the candidate genes survived correction for multiple comparisons at $P<0.05$ in the combined analysis. We note that two genes with the strongest associations in the combined analysis are for genes on chromosome 12 (STAT6 and VDR), which is the chromosome that has often been implicated in linkage studies of asthma (Ober and Hoffjan 2006). STAT6 which was associated with atopic asthma and AHR in this study was recently associated with IgE levels in a genome-wide association study (Weidinger et al. 2008). STAT6, a member of the Signal Transducer and Activator of Transcription (STAT) family acts in signaling pathways linked to IL4 and IL13 and as a transcription factor for genes that are involved in TH2associated processes (Hebenstreit et al. 2006). The Vitamin D Receptor (VDR), as well as Interleukin-13 have been implicated in many genetic association studies (Hebenstreit et al. 2006; Ober and Hoffjan 2006; Poon et al. 2004) and are also involved in $\mathrm{TH} 1-\mathrm{TH} 2$ processes. We found that VDR was associated with all four phenotypes; Poon et al. found significant association with asthma and atopy whereas Raby et al. found association with asthma and $\operatorname{IgE}$ levels in women (Raby et al. 2004). We found two IL13 SNPs (rs2243204 and rs1295686) to be associated with asthma; previous associations for this gene include asthma, atopic asthma, atopy, IgE levels, and AHR. (Ober and Hoffjan 2006; Maier et al. 2006) and is among the most replicated of asthma and allergy genes.

TBXA2R (Thromboxane A2 Receptor) has been extensively studied in the Japanese and Korean populations, but it has not been studied outside of these populations. In these populations, polymorphisms in $T B X A 2 R$ have been associated with asthma, atopy, asprin sensitivity, and blood levels of specific IgE (Ober and Hoffjan 2006; Kim et al. 2008). We provide evidence demonstrating that $T B X A 2 R$ is associated with both atopy and AHR in Caucasians (Table 2). The Thromboxane A receptor, which is involved in prostaglandin and leukotriene pathways, has diverse physiological and pathophysiological actions related to allergies, modulation of acquired immunity, atherogenesis, and neovascularization (Nakahata 2008).

Studying genetic susceptibility variants across diverse studies and populations may increase statistical power when the variants have direct effects on the phenotype that are independent of environment, age, ethnicity, etc. If the genetic variants act in conjunction with other risk factors via interactions (genetic and environmental), they may not be consistently show association across studies. However, providing the results from many studies may be a step toward identification of the environmental and other factors that modulate the expression of the genetic variant.

None of the candidate genes we found to be associated are in regions recently identified in the first genome-wide association study for asthma (Moffatt et al. 2007). Additionally, the current study did not include SNPs in $O R M D L 3$, as this association was reported after the SNPs were selected for this study. We note that the ORMDL3 association has been replicated in the SLSJ cohort (Madore et al. 2008), and numerous other studies (Galanter et al. 2008; Hirota et al. 2008; Tavendale et al. 2008).

This study was limited to only one form of genetic variation, single nucleotide polymorphisms. Although this is likely the major source of variation in the human genome it is becoming increasingly clear that structural alterations including copy number variants and inversions can make a substantial contribution to susceptibility to common diseases (Sharp 2008).

Our study focused on the primary genetic effects, we did not consider the combined additive, or interactive (epistasis) effects of these SNPs, or gene-environment interactions as this was outside the scope of the current work.

In conclusion, this study provides important insight into the interpretation of the literature related to the genetics of asthma and allergy, since many of the genes included have not been extensively studied (Ober and Hoffjan 2006) or have only been studied with small sample sizes $(<300$ subjects, Supplementary Table 1). The present work is an important step in elucidating the genetic etiology of these complex traits. The electronic resource (http://genapha. icapture.ubc.ca/) provides a mechanism for researchers to access and query a large dataset of genetic variation at close to 100 loci, in over 5,000 subjects phenotyped for asthma and allergic traits.

Acknowledgments We thank all subjects who donated time and samples for this project, and numerous health care workers who helped with recruitment and phenotyping. Dr Paul Bégin and Charles Morin for their collaboration and ascertainment of the Saguenay-LacSaint-Jean panel participants. Dr Louis-Philippe Boulet and Michel Laviolette for ascertainment, of the Québec City family trios. For the Canadian Asthma Primary Prevention Study cohort we acknowledge Drs Alexander Ferguson and Wade Watson who phenotyped participants, Ms Roxanne Rousseau and Ms Marilyn Lilley who ascertained subjects and Ms Anne DyBuncio for data management. We gratefully acknowledge the assistance of James Chomin with the TaqMan genotyping of the MTHFR gene, Muriel Grenon for the assistance in recruiting subjects in the Saguenay Lac-St. Jean and Julie Park with the literature search and compiling of Supplementary Table S1. This work was supported by AllerGen, a National Centre of Excellence Network (Canada), the Canadian Institutes of Health Research (CIHR), the Institutes of Gender and Health, BC Lung Association, Génome Québec, and the Respiratory Health Network of the Fonds de la Recherche en Santé du Québec (FRSQ). P.D.P was the recipient of a Michael Smith Foundation Scholarship and the Jacob Churg Distinguished Research Award. T.J.H. received a Clinician-Scientist Award in Translational Research from the Burroughs Wellcome Fund and an Investigator Award from CIHR. The 1994 Busselton follow-up study was funded by Healthway, Western Australia. A.L.J. is a recipient of a National Health and Medical Research Council of Australia Practitioner Fellowship. L.J.P. is supported by 
the National Health and Medical Research Council of Australia and the Wind-Over-Water Foundation. Y. Bossé and D. Daley were recipients of CIHR fellowship awards, and A. Kozyrskyj is a recipient of a New Investigator Award from the Canadian Institutes of Health Research. DD is the recipient of a Michael Smith Foundation Career Scholar Award. C. Laprise, A. Sandford and D. Daley hold Canadian Research Chair appointments.

\section{Appendix: Web resources}

To support further identification and research into the most robust candidate genes for asthma and related phenotypes, we have compiled a comprehensive database that is being made available to the scientific community on our website at http://genapha.icapture.ubc.ca/index.php/research/. This database contains information on all the genes and SNPs studied in the four panels, detailed information on allele frequencies, as well as association results, $P$ values, and linkage disequilibrium (LD) plots for each sample. There are several search tools incorporated into the database, users can search by gene or by SNP. For each SNP there is a comprehensive summary page that gives detailed results for all panels, and phenotypes. The SNP summary page also contains links to dbSNPS, OMIM, and NCBI which will provide users with further information regarding the gene and/or SNP.

Seattle SNPs (http://pga.gs.washington.edu/).

Innate Immunity (http://innateimmunity.net/).

\section{References}

Arshad SH, Hide DW (1992) Effect of environmental factors on the development of allergic disorders in infancy. J Allergy Clin Immunol 90:235-241

Arshad SH, Tariq SM, Matthews S, Hakim E (2001) Sensitization to common allergens and its association with allergic disorders at age 4 years: a whole population birth cohort study. Pediatrics 108:E33

Becker AB, Manfreda J, Ferguson AC, Dimich-Ward H, Watson WT, Chan-Yeung M (1999) Breast-feeding and environmental tobacco smoke exposure. Arch Pediatr Adolesc Med 153:689-691

Becker A, Watson W, Ferguson A, Dimich-Ward H, Chan-Yeung M (2004) The Canadian asthma primary prevention study: outcomes at 2 years of age. J Allergy Clin Immunol 113:650-656

Begin P, Tremblay K, Daley D, Lemire M, Claveau S, Salesse C, Kacel S, Montpetit A, Becker A, Chan-Yeung M, Kozyrskyj AL, Hudson TJ, Laprise C (2007) Association of urokinase-type plasminogen activator with asthma and atopy. Am J Respir Crit Care Med 175:1109-1116

Blakey J, Halapi E, Bjornsdottir US, Wheatley A, Kristinsson S, Upmanyu R, Stefansson K, Hakonarson H, Hall IP (2005) Contribution of ADAM33 polymorphisms to the population risk of asthma. Thorax 60:274-276

Chanock SJ, Manolio T, Boehnke M, Boerwinkle E, Hunter DJ, Thomas G, Hirschhorn JN, Abecasis G, Altshuler D, BaileyWilson JE, Brooks LD, Cardon LR, Daly M, Donnelly P, Fraumeni JF Jr, Freimer NB, Gerhard DS, Gunter C, Guttmacher
AE, Guyer MS, Harris EL, Hoh J, Hoover R, Kong CA, Merikangas KR, Morton CC, Palmer LJ, Phimister EG, Rice JP, Roberts J, Rotimi C, Tucker MA, Vogan KJ, Wacholder S, Wijsman EM, Winn DM, Collins FS (2007) Replicating genotype-phenotype associations. Nature 447:655-660

Chan-Yeung M, Ferguson A, Chan H, Dimich-Ward H, Watson W, Manfreda J, Becker A (1999) Umbilical cord blood mononuclear cell proliferative response to house dust mite does not predict the development of allergic rhinitis and asthma. J Allergy Clin Immunol 104:317-321

Chan-Yeung M, Manfreda J, Dimich-Ward H, Ferguson A, Watson W, Becker A (2000) A randomized controlled study on the effectiveness of a multifaceted intervention program in the primary prevention of asthma in high-risk infants. Arch Pediatr Adolesc Med 154:657-663

Chan-Yeung M, Ferguson A, Watson W, Dimich-Ward H, Rousseau R, Lilley M, Dybuncio A, Becker A (2005) The Canadian childhood asthma primary prevention study: outcomes at 7 years of age. J Allergy Clin Immunol 116:49-55

Dudbridge F (2003) Pedigree disequilibrium tests for multilocus haplotypes. Genet Epidemiol 25:115-121

Dudbridge F (2008) Likelihood-based association analysis for nuclear families and unrelated subjects with missing genotype data. Hum Hered 66:87-98

Galanter J, Choudhry S, Eng C, Nazario S, Rodriguez-Santana JR, Casal J, Torres-Palacios A, Salas J, Chapela R, Watson HG, Meade K, LeNoir M, Rodriguez-Cintron W, Avila PC, Burchard EG (2008) ORMDL3 gene is associated with asthma in three ethnically diverse populations. Am J Respir Crit Care Med 177:1194-1200

Gilliland FD, Berhane K, Li YF, Rappaport EB, Peters JM (2003) Effects of early onset asthma and in utero exposure to maternal smoking on childhood lung function. Am J Respir Crit Care Med 167:917-924

Godfrey S (2000) Bronchial hyper-responsiveness in children. Paediatr Respir Rev 1:148-155

Godfrey S, Springer C, Bar-Yishay E, Avital A (1999) Cut-off points defining normal and asthmatic bronchial reactivity to exercise and inhalation challenges in children and young adults. Eur Respir J 14:659-668

He JQ, Chan-Yeung M, Becker AB, Dimich-Ward H, Ferguson AC, Manfreda J, Watson WT, Sandford AJ (2003) Genetic variants of the IL13 and IL4 genes and atopic diseases in at-risk children. Genes Immun 4:385-389

Hebenstreit D, Wirnsberger G, Horejs-Hoeck J, Duschl A (2006) Signaling mechanisms, interaction partners, and target genes of STAT6. Cytokine Growth Factor Rev 17:173-188

Hegele RG, Ahmad HY, Becker AB, Dimich-Ward H, Ferguson AC, Manfreda J, Watson WT, Chan-Yeung M (2001) The association between respiratory viruses and symptoms in 2-week-old infants at high risk for asthma and allergy. J Pediatr 138:831-837

Hirota T, Harada M, Sakashita M, Doi S, Miyatake A, Fujita K, Enomoto T, Ebisawa M, Yoshihara S, Noguchi E, Saito H, Nakamura Y, Tamari M (2008) Genetic polymorphism regulating ORM1-like 3 (Saccharomyces cerevisiae) expression is associated with childhood atopic asthma in a Japanese population. J Allergy Clin Immunol 121:769-770

International HapMap Consortium (2005) A haplotype map of the human genome. Nature 437:1299-1320

James AL, Knuiman MW, Bartholomew HC, Musk AB (2005a) What can Busselton population health surveys tell us about asthma in older people? Med J Aust 183:S17-S19

James AL, Palmer LJ, Kicic E, Maxwell PS, Lagan SE, Ryan GF, Musk AW (2005b) Decline in lung function in the Busselton health study: the effects of asthma and cigarette smoking. Am J Respir Crit Care Med 171:109-114 
Kaan A, Dimich-Ward H, Manfreda J, Becker A, Watson W, Ferguson A, Chan H, Chan-Yeung M (2000) Cord blood IgE: its determinants and prediction of development of asthma and other allergic disorders at 12 months. Ann Allergy Asthma Immunol 84:37-42

Kim JH, Lee SY, Kim HB, Jin HS, Yu JH, Kim BJ, Kim BS, Kang MJ, Jang SO, Hong SJ (2008) TBXA2R gene polymorphism and responsiveness to leukotriene receptor antagonist in children with asthma. Clin Exp Allergy 38:51-59

Kozyrskyj AL, Mai XM, McGrath P, Hayglass KT, Becker AB, Macneil B (2008) Continued exposure to maternal distress in early life is associated with an increased risk of childhood asthma. Am J Respir Crit Care Med 177:142-147

Laitinen T, Polvi A, Rydman P, Vendelin J, Pulkkinen V, Salmikangas P, Makela S, Rehn M, Pirskanen A, Rautanen A, Zucchelli M, Gullsten H, Leino M, Alenius H, Petays T, Haahtela T, Laitinen A, Laprise C, Hudson TJ, Laitinen LA, Kere J (2004) Characterization of a common susceptibility locus for asthmarelated traits. Science 304:300-304

Laprise C, Sladek R, Ponton A, Bernier MC, Hudson TJ, Laviolette M (2004) Functional classes of bronchial mucosa genes that are differentially expressed in asthma. BMC Genomics 5:21

Li J, Ji L (2005) Adjusting multiple testing in multilocus analyses using the eigenvalues of a correlation matrix. Heredity 95:221227

Li YF, Gilliland FD, Berhane K, McConnell R, Gauderman WJ, Rappaport EB, Peters JM (2000) Effects of in utero and environmental tobacco smoke exposure on lung function in boys and girls with and without asthma. Am J Respir Crit Care Med 162:2097-2104

Lincoln MR, Montpetit A, Cader MZ, Saarela J, Dyment DA, Tiislar M, Ferretti V, Tienari PJ, Sadovnick AD, Peltonen L, Ebers GC, Hudson TJ (2005) A predominant role for the HLA class II region in the association of the MHC region with multiple sclerosis. Nat Genet 37:1108-1112

Madore AM, Tremblay K, Hudson TJ, Laprise C (2008) Replication of an association between 17q21 SNPs and asthma in a FrenchCanadian familial collection. Hum Genet 123:93-95

Mai XM, Becker AB, Sellers EA, Liem JJ, Kozyrskyj AL (2007) Infrequent milk consumption plus being overweight may have great risk for asthma in girls. Allergy 62:1295-1301

Maier LM, Howson JM, Walker N, Spickett GP, Jones RW, Ring SM, McArdle WL, Lowe CE, Bailey R, Payne F, Todd JA, Strachan DP (2006) Association of IL13 with total IgE: evidence against an inverse association of atopy and diabetes. J Allergy Clin Immunol 117:1306-1313

Martinez FD (2007) Gene-environment interactions in asthma: with apologies to William of Ockham. Proc Am Thorac Soc 4:26-31

Melen E, Wickman M, Nordvall SL, van Hage-Hamsten M, Lindfors A (2001) Influence of early and current environmental exposure factors on sensitization and outcome of asthma in pre-school children. Allergy 56:646-652

Moffatt MF, Kabesch M, Liang L, Dixon AL, Strachan D, Heath S, Depner M, von Berg A, Bufe A, Rietschel E, Heinzmann A, Simma B, Frischer T, Willis-Owen SA, Wong KC, Illig T, Vogelberg C, Weiland SK, von Mutius E, Abecasis GR, Farrall
M, Gut IG, Lathrop GM, Cookson WO (2007) Genetic variants regulating ORMDL3 expression contribute to the risk of childhood asthma. Nature 448:470-473

Nakahata N (2008) Thromboxane A2: physiology/pathophysiology, cellular signal transduction and pharmacology. Pharmacol Ther 118:18-35

Nyholt DR (2004) A simple correction for multiple testing for singlenucleotide polymorphisms in linkage disequilibrium with each other. Am J Hum Genet 74:765-769

Ober C, Hoffjan S (2006) Asthma genetics 2006: the long and winding road to gene discovery. Genes Immun 7:95-100

Poon AH, Laprise C, Lemire M, Montpetit A, Sinnett D, Schurr E, Hudson TJ (2004) Association of vitamin D receptor genetic variants with susceptibility to asthma and atopy. Am J Respir Crit Care Med 170:967-973

Poon AH, Laprise C, Lemire M, Hudson TJ, Schurr E (2005) NRAMP1 is not associated with asthma, atopy, and serum immunoglobulin E levels in the French Canadian population. Genes Immun 6:519-527

Raby BA, Klimecki WT, Laprise C, Renaud Y, Faith J, Lemire M, Greenwood C, Weiland KM, Lange C, Palmer LJ, Lazarus R, Vercelli D, Kwiatkowski DJ, Silverman EK, Martinez FD, Hudson TJ, Weiss ST (2002) Polymorphisms in toll-like receptor 4 are not associated with asthma or atopy-related phenotypes. Am J Respir Crit Care Med 166:1449-1456

Raby BA, Lazarus R, Silverman EK, Lake S, Lange C, Wjst M, Weiss ST (2004) Association of vitamin D receptor gene polymorphisms with childhood and adult asthma. Am J Respir Crit Care Med 170:1057-1065

Salam MT, Li YF, Langholz B, Gilliland FD (2004) Early-life environmental risk factors for asthma: findings from the Children's health study. Environ Health Perspect 112:760-765

Sharp AJ (2008) Emerging themes and new challenges in defining the role of structural variation in human disease. Hum Mutat. (Epub ahead of print)

Shen R, Fan JB, Campbell D, Chang W, Chen J, Doucet D, Yeakley J, Bibikova M, Wickham Garcia E, McBride C, Steemers F, Garcia F, Kermani BG, Gunderson K, Oliphant A (2005) Highthroughput SNP genotyping on universal bead arrays. Mutat Res 573:70-82

Tavendale R, Macgregor DF, Mukhopadhyay S, Palmer CN (2008) A polymorphism controlling ORMDL3 expression is associated with asthma that is poorly controlled by current medications. J Allergy Clin Immunol 121:860-863

Tremblay K, Lemire M, Provost V, Pastinen T, Renaud Y, Sandford AJ, Laviolette M, Hudson TJ, Laprise C (2006) Association study between the CX3CR1 gene and asthma. Genes Immun 7:632-639

Weidinger S, Gieger C, Rodriguez E, Baurecht H, Mempel M, Klopp $\mathrm{N}$, Gohlke H, Wagenpfeil S, Ollert M, Ring J, Behrendt H, Heinrich J, Novak N, Bieber T, Kramer U, Berdel D, von Berg A, Bauer CP, Herbarth O, Koletzko S, Prokisch H, Mehta D, Meitinger T, Depner M, von Mutius E, Liang L, Moffatt M, Cookson W, Kabesch M, Wichmann HE, Illig T (2008) Genome-wide scan on total serum IgE levels identifies FCER1A as novel susceptibility locus. PLoS Genet 4:e1000166 